\title{
HOLOMORPHIC DIFFERENTIALS AS FUNCTIONS OF MODULI ${ }^{1}$
}

\author{
LIPMAN BERS \\ Communicated November 21, 1960
}

The purpose of this note is to strengthen the results of [3] and to indicate a very brief derivation of some theorems announced without proof in $[1 ; 3]$.

We begin by indicating a correction to [3]. Let $S_{1}$ and $S_{2}$ be Riemann surfaces, $f$ an orientation preserving (orientation reversing) homeomorphism of bounded eccentricity of $S_{1}$ onto $S_{2}$ and $[f]$ the homotopy class of $f$; then $\left(S_{1},[f], S_{2}\right)$ is called an even (odd) coupled pair of Riemann surfaces. The definition of equivalence of such pairs given in [3] is imprecise and garbled by misprints. The correct definition reads: $\left(S_{1},[f], S_{2}\right)$ and $\left(S_{1}^{\prime},\left[f^{\prime}\right], S_{2}^{\prime}\right)$ are called equivalent if there exist conformal homeomorphisms $h_{1}$ and $h_{2}$ with $h_{1}\left(S_{1}\right)=S_{1}^{\prime}$, $h_{2}\left(S_{2}\right)=S_{2}^{\prime}$ and $\left[h_{2} f\right]=\left[f^{\prime} h_{1}\right]$; the two pairs are called strongly equivalent if $S_{2}^{\prime}=S_{2}$ and there exists a conformal homeomorphism $h$ with $h\left(S_{1}\right)=S_{1}^{\prime}$ and $[f]=\left[f^{\prime} h\right]$. If $S_{0}$ is a Riemann surface, then the Teichmüller space $T\left(S_{0}\right)$ can be thought of as the set of strong equivalence of even pairs $\left(S,[f], S_{0}\right)$ (and not of simple equivalence classes as stated in [3]). ${ }^{2}$

From now on we assume that $S_{0}$ is a fixed closed Riemann surface of genus $g>1$, and we write $T$ instead of $T\left(S_{0}\right)$. $T$ has a natural complex analytic structure and can be represented as a bounded domain, homeomorphic to a ball, in complex number space with coordinates (moduli) $\tau_{1}, \cdots, \tau_{3 g-3}$ (cf. $[1 ; 2]$ ). Points of $T$ will be denoted by $\tau$. We may assume that $S_{0}$ is given as the unit disc modulo a fixedpoint-free Fuchsian group, and that $\tau=0$ corresponds to the pair $\left(S_{0}\right.$, [identity], $\left.S_{0}\right)$.

TheOREM I. One can associate to every $\tau \in T$ a bounded Jordan domain $D(\tau)$ and $2 g$ Möbius transformations $z \rightarrow A_{j}(z, \tau), z \rightarrow B_{j}(z, \tau)$, $j=1, \cdots, g$, such that the following conditions are satisfied.

1 This paper represents results obtained at the Institute of Mathematical Sciences, New York University, under the sponsorship of the Office of Ordnance Research, U. S. Army, Contract No. DA-30-069-ORD-2153. Reproduction in whole or in part permitted for any purpose of the United States Government.

2 We also note the following errata to $[2 ; 3]$. On p. $94,1.19$, replace $(\zeta)$ by $\mu(\zeta)$. On p. $96,1.15$, replace the subscript $j$ by $2 j$. On p. $97,1.21$, replace $C_{r}$ by $C^{r}$. On p. 100 , 1. 4 , replace 'covering' by 'covering space.' On p. 103, equation (9) replace the exponent $3 g-3 n+n$ by $3 g-3+n$. 
(i) The boundary curve of $D(\tau)$ admits the parametric representation $z=\sigma\left(e^{i \theta}, \tau\right), 0 \leqq \theta \leqq 2 \pi$, depending holomorphically on $\tau$. tion

(ii) The $A_{j}$ and $B_{j}$ depend holomorphically on $\tau$ and satisfy the rela-

$$
\prod_{j=1}^{0} A_{j} B_{j} A_{j}^{-1} B_{j}^{-1}=1 .
$$

For every fixed $\tau \in T$ they generate, with the single defining relation (1), a fixed-point-free discrete group $G(\tau)$ of conformal self-mappings of $D(\tau)$, so that $S(\tau)=D(\tau) / G(\tau)$ is a closed Riemann surface of genus $g$. $S(0)$ is the surface $S_{0}$.

(iii) Denote by $\alpha(\tau)$ the basis of the fundamental group of $S(\tau)$ defined by $A_{1}, \cdots, B_{g}$, and by $f_{\tau}$ a quasiconformal mapping of $S(\tau)$ onto $S(0)$ which takes $\alpha(\tau)$ into $\alpha(0)$. Then the point $\tau$ corresponds to the pair $\left(S(\tau),\left[f_{\tau}\right], S_{0}\right)$.

This statement differs from Theorem 2 in [3] primarily by the boundedness condition for $D(\tau)$ and can be obtained from that theorem without much difficulty.

We denote by $M$ the domain in complex number space of $3 g-2$ dimensions which consists of points $(z, \tau)$ with $z \in D(\tau)$ and $\tau \in T$. By Theorem 3 in [3] $M$ is holomorphically equivalent to $T\left(S_{0}-\{p\}\right)$ for a fixed $p \in S_{0}$.

We denote by $W_{q}(\tau)$ the (complex) vector space of holomorphic functions $\phi(z), z \in D(\tau)$, for which $\phi(z) d z^{q}$ is invariant under $G(\tau)$; this is the same as the space of $q$-dimensional holomorphic differentials on $S(\tau)$, so that $\operatorname{dim} W_{q}(\tau)=0,1, g$, or $(2 q-1)(g-1)$ according to whether $q<0, q=0, q=1$, or $q>1$. In $W_{1}(\tau)$ there exist $g$ distinguished elements, $p_{k}(z, \tau)$, determined by the conditions

$$
\int_{z}^{A_{i}(z, \tau)} p_{k}\left(z^{\prime}, \tau\right) d z^{\prime}=\delta_{i k} ;
$$

these correspond to the normalized Abelian differentials of the first kind on $S(\tau)$ belonging to the "canonical" homology basis $a(\tau)$ determined by $\alpha(\tau)$. The period matrix of $S(\tau)$ belonging to $a(\tau)$ will be denoted by $Z(\tau)$. It has the elements

$$
Z_{i k}(\tau)=\int_{z}^{B_{i}(z, \tau)} p_{k}\left(z^{\prime}, \tau\right) d z^{\prime}
$$

and is a point in the Siegel space of symmetric matrices with positive definite imaginary part. 
We denote by $W_{q}$ the vector space of holomorphic functions $\Phi(z, \tau)$, $(z, \tau) \in M$, which belong to $W_{q}(\tau)$ for every fixed $\tau \in T$.

THEOREM II. Every element of $W_{q}(\tau)$ is a restriction of an element of $W_{q}$.

Proof. Assume that $q \geqq 2$. Let $C_{j}, j=1,2, \cdots$, be a complete system of nonequivalent (with respect to (1)) words in the letters $A_{1}, \cdots, B_{0}$. If $P(t)$ is a polynomial, then the Poincaré series

$$
\sum_{j=1}^{\infty} P\left(C_{j}(z, \tau)\right)\left(\partial C_{j}(z, \tau) / \partial z\right)^{q}
$$

converges normally in $M$ and its sum belongs to $W_{q}$. On the other hand, since $D(\tau)$ is a bounded Jordan domain and $G(\tau)$ has a compact fundamental region, Theorem 4 in [4] implies that, for a fixed $\tau$, every element of $W_{q}(\tau)$ is of the form (3).

For $q=1$ we shall show that every $p_{j}$ belongs to $W_{1}$ (i.e., that the normalized Abelian differentials are holomorphic functions of the moduli).

TheOREM III. The functions $p_{k}(z, \tau), k=1, \cdots, g$, are holomorphic in $M$.

Proof. It suffices to consider $p_{1}$. We shall show that in a neighborhood of a fixed but arbitrary point $\tau_{0} \in T$ we have an identity of the form

$$
p_{1}(z, \tau)=\Phi(z, \tau)^{-1} \sum_{j=1}^{5 g-5} c_{j}(\tau) \Phi_{j}(z, \tau)
$$

where the $c_{j}$ are holomorphic, $\Phi \in W_{2}$, and the $\Phi_{j}$ are elements of $W_{3}$. We first choose $\Phi$ so that $\Phi\left(z, \tau_{0}\right)$ vanishes at $4 g-4$ points $z_{i}$ which are not equivalent under $G\left(\tau_{0}\right)$. This is possible since the "general" holomorphic quadratic differential on $S(\tau)$ has only simple zeros (Bertini) and hence exactly $4 g-4$ of those. There exist $4 g-4$ holomorphic functions $\zeta_{i}(\tau)$ defined near $\tau_{0}$, such that $\zeta_{i}\left(\tau_{0}\right)=0$ and $\Phi\left(z_{i}+\zeta_{i}(\tau), \tau\right)$ $=0$. In order that the right hand side of $(4)$ belong to $W_{1}(\tau)$ it is necessary and sufficient that

$$
\sum_{j=1}^{5 o-5} c_{j}(\tau) \Phi_{j}\left(z_{i}+\zeta_{i}(\tau), \tau\right)=0, \quad i=1, \cdots, 4 g-4,
$$

and one sees at once that any $4 g-5$ of these equations imply the $(4 g-4)$ th. In order that $(4)$ hold near $\tau_{0}$ the $c_{j}$ must satisfy $g$ additional linear equations which are obtained from (1) by setting $k=1$ 
and choosing a fixed point $z$ and fixed paths of integration, avoiding the points $z_{i}$. The resulting linear system, with holomorphic coefficients, for the unknown functions $c_{j}$, is uniquely solvable at $\tau_{0}$ if the functions $\Phi_{1}, \cdots, \Phi_{b_{g-5}}$ are chosen so as to be linearly independent for $\tau=\tau_{0}$. In this case the equations are also uniquely solvable for $\tau$ close to $\tau_{0}$, and the solutions depend holomorphically on $\tau$.

We proceed to derive some consequences from Theorems II and III.

(a) The functions

$$
f_{i j}=p_{i} / p_{j}, \quad f_{i j k}=f_{k}^{-1} \partial \log f_{i j} / \partial z
$$

are meromorphic in $M$. This proves Theorem $\mathrm{J}$ in [1]. It is classical that every meromorphic function in $D(\tau)$ which is automorphic under $G(\tau)$ can be expressed rationally in terms of the functions $f_{i j}, f_{i j k}$ (and even in terms of the $f_{i j}$ alone if $S(\tau)$ is not hyperelliptic). Thus we obtain a proof of Theorem 4 in [3] which asserts the existence of finitely many meromorphic functions of the moduli and of an additional complex variable, which uniformize simultaneously all algebraic curves of genus $g>1$.

(b) Let us choose $(2 q-1)(g-1)$ elements of $W_{q}, q>1$ (or $g$ elements of $\left.W_{1}\right)$ which are linearly independent for $\tau=\tau_{0}$, and let $w(z, \tau)$ denote their Wronskian with respect to $z$. For a fixed $\tau$ close to $\tau_{0}$ the zeros of $w(z, \tau)$ are precisely the Weierstrass points of $S(\tau)$, in the classical sense if $q=1$, in the sense of Petersson if $q>1$ (cf. the definition in [4]). Since $w$ is a holomorphic function in $M$ we conclude that the Weierstrass points on a closed Riemann surface depend holomorphically on the moduli (cf. Rauch [6], Röhrl [3]).

(c) Now let $w(z, \tau)$ denote the Wronskian of an arbitrary set of $\operatorname{dim} W_{q}(\tau)$ elements of $W_{q}$ and let $N$ denote the set of those $\tau \in T$ for which $w(z, \tau) \equiv 0$. If $z_{0}$ is not a Weierstrass point of $S\left(\tau_{0}\right)$, then there is a neighborhood of $\tau_{0}$ in which the points of $N$ are precisely the zeros of $w\left(z_{0}, \tau\right)$. We conclude that $N$ is either empty, or the whole domain $T$, or an analytic subset of $T$ of codimension 1 .

(d) Let $H$ denote the set of those $\tau \in T$ for which $S(\tau)$ is hyperelliptic. For $\tau \in T-H$ every element of $W_{q}(\tau)$ can be written as a homogeneous polynomial in the $p_{j}$ (M. Noether). For $\tau \in H$ the subspace of $W_{q}(\tau)$ consisting of homogeneous polynomials in elements of $W_{1}(\tau)$ has dimension $q(g-1)+1$. But $H$ is an analytic subvariety of $T$ of dimension $2 g-1$, so that, noting (c), we obtain the following complement to Noether's theorem: for $g>3$ and $q>1$ there exist no fixed set of $(2 q-1)(g-1)$ homogeneous polynomials of degree $q$ in normalized Abelian differentials of the first kind which spans the space of 
holomorphic differentials of dimension $q$ on all nonhyperelliptic closed Riemann surfaces of genus $g$.

(e) The mapping $\tau \rightarrow Z(\tau)$ of the Teichmüller space into the Siegel space is holomorphic. This follows at once from Theorem III, and also by using the coordinates in $T$ defined in [1] in conjunction with Rauch's variational formulas [5]. These formulas also show that the mapping of $T$ into a $(3 g-3)$-dimensional subspace of the Siegel space

$$
\tau \rightarrow\left\{\sum_{i, k=1}^{g} \gamma_{j, i k} Z_{i k}(\tau), j=1, \cdots, 3 g-3\right\}
$$

is one-to-one near a point $\tau_{0}$ if and only if the $3 g-3$ functions

$$
\sum_{i, k=1}^{g} \gamma_{j, i k} p_{i}\left(z, \tau_{0}\right) p_{k}\left(z \quad \tau_{0}\right)
$$

are linearly independent. This shows that near every nonhyperelliptic surface a properly chosen set of $3 g-3$ periods $Z_{i k}$ can serve as a set of local moduli (Rauch). On the other hand, (d) implies a complement to Rauch's theorem: no fixed set of $3 g-3$ linear combinations of periods can serve as a set of moduli near every nonhyperelliptic closed Riemann surface of genus $\mathrm{g}>3$.

\section{REFERENCES}

1. Lipman Bers, Spaces of Riemann surfaces, Proceedings of the International Congress of Mathematicians, Edinburgh, 1958, pp. 349-361.

2. - Spaces of Riemann surfaces as bounded domains, Bull. Amer. Math. Soc. vol. 66 (1960) pp. 98-103.

3. - Simultaneous uniformization, Bull. Amer. Math. Soc. vol. 66 (1960) pp. 94-97.

4. - Completeness theorems for Poincaré series in one variable, to appear.

5. H. E. Rauch, On the transcendental moduli of algebraic Riemann surfaces, Proc. Nat. Acad. Sci. U.S.A. vol. 41 (1955) pp. 42-49.

6. - Weierstrass points, branch points, and moduli of Riemann surfaces, Comm. Pure Appl. Math. vol. 13 (1959) pp. 543-560.

7. Helmut Röhrl, On holomorphic families of fiber bundles over the Riemann sphere, to appear.

New York University 